\title{
Religion, die vergessene Differenz
}

\section{Ulrich Krainz}

Online publiziert: 23. Mai 2015

(C) Springer Fachmedien Wiesbaden 2015

Die vorliegende Ausgabe der Zeitschrift Gruppendynamik und Organisationsberatung hat während des Schreibprozesses , unbeabsichtigt' an Relevanz gewonnen. Die Vorbereitungen zu diesem Themenband waren begleitet von gesellschaftspolitischen Ereignissen und Aktionen, die stets im Namen der Religion gesetzt wurden und durch ihre Archaik und Destruktivität erschrecken - sei es die gewaltsame Ausbreitung unterschiedlicher islamistischer Terrorgruppen wie Boko Haram in Nigeria, der AlShabaab-Milizen in Somalia und Kenia, das blutige Vorgehen der Terrormilizen des ,Islamischen Staats' (IS) in Syrien und dem Irak, das auch radikal-fundamentalistische Muslime aus aller Welt anzieht, oder die Terroranschläge in Europa wie z. B. auf das französische Satiremagazin Charlie Hebdo. Es ist daher nicht verwunderlich, dass diese aktuellen Ereignisse auch in manchen Beiträgen dieses Themenhefts wenn auch nicht immer explizit fokussiert - anklingen.

Das Themenheft ist somit ,am Puls der Zeit‘ und nimmt sich eines höchst aktuellen, jedoch wenig konsequent verfolgten Themas an. Religion als ein expliziter Untersuchungsgegenstand stellt in der wissenschaftlichen Diskussion eher die Ausnahme als die Regel dar. Zwar werden solche Diskurse vor allem im Kontext der Religionssoziologie, Kultur- oder Politikwissenschaft geführt, die Überlegungen sind aber auch für die Gruppendynamik als anwendungsorientierte Sozialwissenschaft und im Weiteren für die Organisationsberatung von Bedeutung. Die jüngsten Ereignisse unterstreichen die Wichtigkeit - ja Notwendigkeit - sich fundierter mit dem Thema Religion und den Auswirkungen religiös-sinnstiftender Systeme auf soziale Prozesse auseinanderzusetzen, sei es in Gruppen, Organisationen oder der Gesellschaft als ganzer.

Mag. Dr. U. Krainz (ه)

Zentrum für LehrerInnenbildung (ZLB),

Universität Wien,

Porzellangasse 4 ,

1090 Wien, Österreich

E-Mail: ulrich.krainz@univie.ac.at 
Das ,Funktionieren“ von Gesellschaft ist ohne die wirksame Gestaltungskraft von Religion für die Lebenspraxis vieler Menschen nur unzureichend interpretierbar. Auch für die Trainings- und Beratungsarbeit ist der Faktor Religion von Bedeutung, gleichviel ob man an religiös homogene oder heterogene Kontexte (durchmischt mit anscheinend areligiösen Anteilen) denkt. Moderne Unternehmen und Organisationen unterliegen heute zahlreichen gesellschaftlichen Veränderungsdynamiken. Migrations-, Globalisierungs- und Internationalisierungsprozesse führen dazu, dass immer mehr gemischtkulturelle Arbeits- und Lebenszusammenhänge entstehen. Das damit einhergehende Aufeinandertreffen (um nicht zusagen Zusammenprallen) unterschiedlicher Kulturen gibt somit zu Reflexionsbemühungen Anlass, die auch die Religion und ihre jeweiligen Transformationsprozesse berücksichtigen muss. Mit dem Programm der Aufklärung, die als ,Mega-Projekt' die Moderne in Europa mitbegründet hat, hat auch die Religion einen zentralen Bedeutungswandel erfahren. In unterschiedlichem Ausmaß kam es zu einem Säkularisierungsprozess, einem Zurückdrängen des Einflusses institutionalisierter Kirchen und Religionsgemeinschaften auf das gesellschaftlich-öffentliche Leben und einer Verlagerung des Religiösen ins individuell Private. In zweierlei Hinsicht ist dieser Zustand relativ. Zum einen lässt sich säkularisiertes Bewusstsein nicht einfach auf andere Weltgegenden übertragen; zum anderen werden die (zum Teil hart erkämpften) säkularisierten Lebensformen durch die Einwanderung von Menschen aus Kulturen, die keinen Säkularisierungsund Aufklärungsprozess durchlaufen haben, und durch das beobachtbare Revival streng orthodoxer religiöser Glaubenssysteme einem Bestandstest unterzogen. Nicht wenige entdecken ihre eigene ,Fundamentalisierungsbereitschaft ${ }^{\star}$ und scheinen ihr zu erliegen (vgl. das in der Bundesrepublik Deutschland aufgekommene Wort ,Leitkultur' und die rechtspopulistischen Slogans eines ,Wehrhaften Christentums` etc.).

Es ist daher durchaus angebracht sich diesen gesellschaftlichen Makroströmungen, die längst über nationale Grenzen hinausgehen, zu widmen und sich der Folgen auf Mikroebene anzunehmen. Interessant für das Verständnis und die Handhabung sozialer Situationen (im Sinne von Gruppendynamik und Organisationsberatung) ist, dass diese Fragestellungen stets in konkreten Interaktionssituationen auftauchen. Die Pluralisierung der Gesellschaft und der Weg in eine, Weltgesellschaft' bringen also nicht einfach nur multikulturelle, sondern zusehends auch multireligiöse Konstellationen hervor. Es kommt zu einem gleichzeitigen Zusammenleben und Aufeinandertreffen unterschiedlicher religiöser sowie säkularer Ansichten und Weltanschauungen, einem Zusammenfließen ,nicht gleichsinnig Denkender', was potentiell konfliktbehaftete Situationen konstituiert. Soll man sie ignorieren? Sie vermeiden? Sie diskursiv bearbeiten? Sie ,professionell begleiten'? Wie? Auch scheint es, dass Multikulturalität als Begriff weit weniger ,Dramatik` enthält als der Begriff Multireligiosität. In der Rückbesinnung auf Kurt Lewin ist die Bearbeitung von Unterschieden immerhin zentraler Ausgangspunkt bei der „Lösung sozialer Konflikte“ (Lewin 1948). Dass Religion als Differenzmerkmal jedenfalls in die Überlegungen mit aufzunehmen ist, dafür plädieren die Beiträge in diesem Heft. Alle Bemühungen um ,Verständnis ‘ und Verständigung implizieren die Frage nach Differenzbewältigung und ihre Bedeutung für soziales Handeln.

Aber auch innerhalb als säkular bezeichneter Gesellschaften lassen sich Spuren des Religiösen an unterschiedlichen Stellen nachzeichnen. Dies beginnt etwa in profes- 
sionellen Beratungstätigkeiten wie Coaching und Supervision, die - obwohl säkular ausgerichtet und konzipiert - immer wieder existentielle Fragen und eine Sinnsuche tangieren, die üblicherweise mit Religion oder ,Spiritualität' assoziiert werden. Die Rede von der so genannten ,charismatischen Führung' nimmt genauso religiöse Züge an, wie sinnstiftend gemeinte Maßnahmen innerhalb von Organisationen (Code of Conduct, Leitbilder und Symbole etc.), die in ihrer intendierten Wirksamkeit für Organisationsmitglieder wie religiöse Glaubensbekenntnisse anmuten. Religiöse Aufladungen zeigen sich - für viele überraschend - auch in jenem Bereich, der vorgeblich als der Rationalste gilt, in der Geldökonomie. Die mit ihr verbundenen Handlungsweisen, wie etwa das Streben nach Geld in der kapitalistischen Welt (die ,Anbetung des Gottes Mammon'), das Aufnehmen von Schulden (und ihre jeweilige Erlassung) zeigen sich bei genauerer Betrachtung ebenfalls als in religiösem Gewand eingekleidet.

Die Beiträge des Heftes betrachten dieses Thema mit unterschiedlichen Fokusbildungen. Der einleitende Artikel von Ulrich Krainz widmet sich dem grundsätzlichen Konfliktpotential religiöser Glaubenssysteme und den möglichen Folgen für die Arbeit in und mit Organisationen. Obwohl das Thema Religion bzw. religiöse Differenz innerhalb wie außerhalb von Organisationen zunehmend sichtbarer und bedeutsamer wird, fällt auf, dass dieses in den die Beratungsliteratur kennzeichnenden Diskursen zur ,Interkulturellen Kompetenz' bzw. des ,Diversity Managements“ nicht immer seine entsprechende Berücksichtigung findet. In Auseinandersetzung mit kulturanthropologischen und kulturtheoretischen Überlegungen wird daher für eine Bewusstseinsbildung und eine reflexive Beschäftigung mit dem Thema Religion und ihren sozialinteraktiven Folgen plädiert, was eine notwendige Aufmerksamkeitserweiterung für trainings- und beratungsbezogenes Arbeiten erfordert.

Die Frage des Umgangs mit dem Thema Religion in Beratungszusammenhängen greift der Beitrag von Adelheid Fiedler auf. Wie kann bzw. soll man mit religiösen Themen und Haltungen umgehen, wenn sie in der Beratung zur Sprache kommen, man als beratende Person vielleicht keinen religiösen Erfahrungshintergrund bzw. entsprechende Überzeugungen teilt, das Religiöse womöglich überhaupt befremdet? Um nicht abwertenden Denkschemata zu erliegen, die dichotomen Unterscheidungen folgen, wie etwa religiös vs. modern, fromm vs. vernünftig, gläubig vs. aufgeklärt usw., braucht es einen entsprechenden Begegnungsraum, weshalb eine metareligiöse, ihrer Natur nach säkulare Ebene einzunehmen ist. Dass die dadurch charakterisierte Beratungshaltung auch eine Selbstreflexion auf Seiten professionell Beratender erfordert, wird in dem Beitrag ausgeführt.

Mit dem Verhältnis von Religion und Gruppendynamik, insbesondere der Praxis in gruppendynamischen Trainingsgruppen, beschäftigen sich gleich zwei Beiträge.

In Form einer philosophisch-anthropologischen Reflexion sind Peter Heintel und Ruth Erika Lerchster in ihrem Beitrag von der Frage geleitet, in wieweit ein Zusammenhang zwischen dem Christentum - als trinitarische Religion - und der gruppendynamischen Praxis hergestellt werden kann. So erscheint gerade in Zeiten der ,Hierarchiekrise', in der man sich zunehmend von allwissenden Autoritäten verabschieden muss, die gruppendynamische Praxis einen wesentlichen Beitrag zur ,kollektiven Selbsttranszendenz' in Organisationszusammenhängen zu leisten, wobei die christliche Trinität als Möglichkeit angesehen wird, sich aus solchen Abhängigkeiten $\mathrm{zu}$ (er)lösen. 
Gerhard Schwarz diskutiert in seinem Beitrag den Gedankengang, wie sich die Entwicklung der Religion in der Form gruppendynamischer T-Gruppen widerspiegelt. In der Tradition der Aufklärung und im Anschluss an die Erlösungsreligionen stehend, werden mit Hilfe der Methode der Gruppendynamik Lernprozesse in Gruppen initiiert, die auf eine Analyse von Abhängigkeiten und eine Einbeziehung von Widersprüchen abzielen und schließlich zu gemeinsamen Konsenslösungen führen. Mit dem Gedanken der ,Erlösung ‘ als ein zu-sich-Kommen des Menschen mit allen Konsequenzen für eine verantwortliche Lebensführung und der Übersetzung des christlichen Liebesgebots als Bemühung um Konsens - eine Bemühung, die ihrerseits bedeutet, dass man sich auf keine Autoritäten verlassen kann - wird in dem Beitrag aufgezeigt, welche religiösen Dimensionen ein derartiges gruppendynamisches Vorgehen aufweist.

Der Beitrag von Karin Lackner widmet sich zunächst den Veränderungen der Arbeitswelt und thematisiert dabei unterschiedlichste Mobilitäts- und Flexibilitätszumutungen, mit denen sich MitarbeiterInnen in Unternehmen heutzutage konfrontiert sehen. Die Freisetzung des Individuums und der damit einhergehende Verlust von Bindungen und Zugehörigkeitsgefühlen zur jeweiligen Organisation (Affiliationsverlust), der als Folge ständiger Mobilitätsbereitschaft, Internationalisierung und nur kurzfristiger Eingebundenheit entsteht, stellt Personalentwicklungsabteilungen vor große Herausforderungen. Wie können MitarbeiterInnen im Unternehmen gehalten werden? Wie kann man sich als Arbeitgeber attraktiv machen? In ihrem Bemühen Bindung herzustellen, zeigen sich zahlreiche organisationale Kompensationen, unterschiedlichste identitäts- und sinnstiftende Maßnahmen, die bei genauerer Betrachtung wie spirituelle bzw. quasi-religiöse Inszenierungen erscheinen.

Der letzte Beitrag von Eva-Maria Lauckner nimmt Bezug auf ein Forschungsprojekt, das sich der Erforschung von Fremdheit bei deutschen Expatriates in Syrien (noch vor den gegenwärtigen kriegerischen Auseinandersetzungen) angenommen hat. Dabei wird diskutiert, mit welchem mitgebrachten ,mentalen Reisegepäck ‘ in Bezug auf das Thema Religion die entsandten Personen auf ihre neuen (muslimischen) KollegInnen treffen, und dass festschreibende Meinungsbilder über ,den Islam' Stereotypen und Abgrenzungsmechanismen befördern. Vor dem Hintergrund dieser Überlegungen erscheinen geeignete Vorbereitungsmaßnahmen für Entsandte mehr als sinnvoll.

Dass das Thema Religion im Kontext von Gruppendynamik und Organisationsberatung so lange unberücksichtigt geblieben ist, verwundert, handelt es sich bei Religiosität doch um ein - man möchte fast sagen - anthropologisches Merkmal (Luckmann 1967), das sich als Phänomen überall auf der Welt und in unterschiedlichen Kontexten beobachten lässt. Mit der expliziten Fokussierung auf das Thema Religion und seine Bedeutung für Gruppendynamik und Organisationsberatung wollen wir damit beginnen eine Leerstelle aufzufüllen, die im Grunde ein weites Themenspektrum umfasst. Es ist wohl nicht allzu ,prophetisch' gedacht, zukünftig mit einer Verstärkung der hier eingeschlagenen Aufmerksamkeitsrichtung zu rechnen.

Der interessierten Leserschaft wünschen wir Anregungen zum Weiterdenken. Ulrich Krainz 


\section{Literatur}

Lewin, K. (1948). Resolving social conflicts. Selected papers on group dynamics. New York: Harber \& Row.

Luckmann, T. (1967). The invisible religion. The problem of religion in modern society. New York: Mc Millan. 